\title{
ERRATUM
}

\author{
Zhuoming XU $\cdot$ Xiao CAO $\cdot$ Yisheng DONG \\ Yahong HAN
}

\section{s-HITSc: an improved model and algorithm for topic distillation on the Web}

Published online: 15 October 2005

(C) Springer-Verlag 2005

\section{Soft Comput (2006) 10:2-11}

Unfortunately, the paper s-HITSc: an improved model and algorithm for topic distillation on the Web by Zhuoming XU, Xiao CAO, Yisheng DONG and Yahong HAN (DOI 10.1007/s00500-005-0457-0) was published with errors. The correct sections are given here:

Section 2.1: I operation: $\mathbf{a}[u]:=\sum_{\{v \mid(v, u) \in E\}} \mathbf{h}[v]$

Section 2.1: $\boldsymbol{O}$ operation: $\mathbf{h}[u]:=\sum_{\{v \mid(u, v) \in E\}} \mathbf{a}[v]$.

Section 3.2: Definition 1 (site $s_{Q}$ ): A site

Section 3.2: Definition 2 (site base-set $\mathcal{B}_{Q}$ ): A site base-set $\mathcal{B}_{Q}$
Section 3.2:

Algorithm Aggregation $\left(B_{Q}\right)$

//Algorithm for forming a site base-set.

$B_{Q}=\left\{p_{1}, p_{2}, \cdots, p_{n}\right\}:$ a page base-set containing $n$

pages.

1. $m:=1 ; C_{l}:=\left\{p_{l}\right\} ; i d_{l}:=p_{l}$.

2. FOR $i:=2 \mathrm{TO} n \mathrm{DO}$

3. [ GET a page $p_{i} \in B_{Q}$, and set put :=FALSE.

4. $\quad$ FOR $j:=1 \mathrm{TO} m$ DO

5. IF URL_host $\left(p_{i}\right)=\mathbf{U R L} \_\operatorname{host}\left(i d_{j}\right)$ THEN

6. $\quad\left[C_{j}:=C_{j} \mathrm{U}\left\{p_{i}\right\}\right.$.

7. IF URL_depth $\left(p_{i}\right)<$ URL_depth $\left(i d_{j}\right)$

THEN $i d_{j}:=p_{i}$.

8. Set $p u t:=$ TRUE, then EXIT from the $j$ loop. ].

9. IF NOT put THEN $\left[m:=m+1 ; C_{m}:=\left\{p_{i}\right\}\right.$;

$$
\left.i d_{m}:=p_{i} \cdot\right] \text {. ]. }
$$

10. Set sites $s_{Q}^{1}:=\left(i d_{1}, C_{1}\right), s_{Q}^{2}:=\left(i d_{2}, C_{2}\right), \ldots$,

$$
\begin{aligned}
& s_{Q}^{m}:=\left(i d_{m}, C_{m}\right), \text { and site base-set } \\
& \mathcal{B}_{Q}:=\left\{s_{Q}^{1}, s_{Q}^{2}, \ldots, s_{Q}^{m}\right\} .
\end{aligned}
$$

11. RETURN $\mathcal{B}_{Q}$.

The online version of the original article can be found at http:// dx.doi.org/10.1007/s00500-005-0457-0

Z. XU · Y. DONG

Department of Computer Science and Engineering,

Southeast University, Nanjing, 210096, China

E-mail: ysdong@seu.edu.cn

Z. XU $(\bowtie) \cdot$ X. CAO $\cdot$ Y. HAN

College of Computers and Information Engineering,

Hohai University, Nanjing, 210098, China

E-mail: zmxu@acm.org

E-mail: xcao@ieee.org

E-mail: hanyahong@hotmail.com 
Section 3.3:

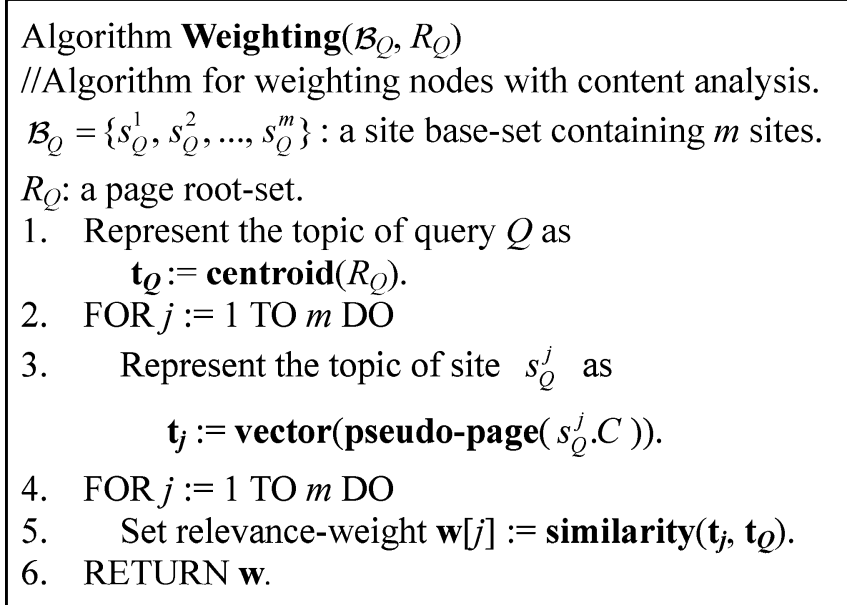

Section 3.4: $\boldsymbol{I}_{w}$ operation: $\mathbf{a}[u]:=\sum_{\{v \mid(v, u) \in E\}}(\mathbf{w}[v] \times \mathbf{h}[v])$

Section 3.4 Algorithm SGraph:

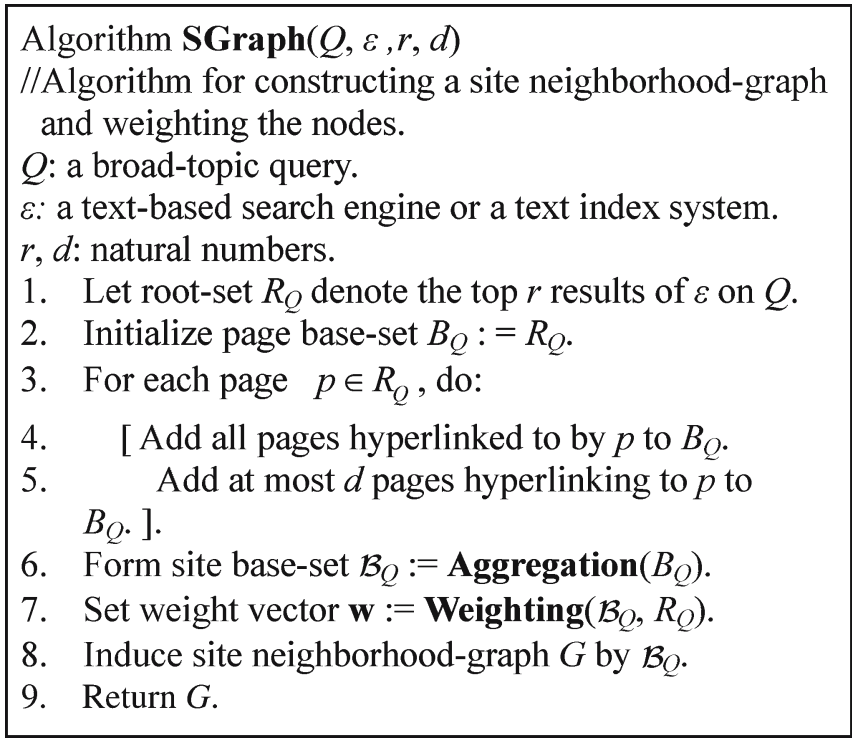

Section 3.4 Algorithm Witeration:

Algorithm WIteration $(G, k, c)$

//Algorithm for reporting top-ranked authorities \& hubs.

$k, c$ : natural numbers.

1. Initialize vectors $\mathbf{a}$ and $\mathbf{h}$ to $\mathbf{z}=(1,1, \ldots, 1)^{T} \in R^{n}$.

2. FOR $i:=1$ TO $k \mathrm{DO}$

3. [Apply the $I_{w}$ operation on $\mathbf{h}$ to compute $\mathbf{a}$.

4. Apply the $\boldsymbol{O}_{\boldsymbol{w}}$ operation on a to compute $\mathbf{h}$.

5. Normalize $\mathbf{a}$ and $\mathbf{h}$ to unit vectors. ].

6. Report the nodes with the $c$ largest coordinates in vector a as authorities, and the nodes with the $c$ largest coordinates in vector $\mathbf{h}$ as $h u b s$, respectively.

References 6: 6. Craswell N, Hawking D, Robertson S (2001) Effective site finding using link anchor information. In: Proceedings of the 24th annual international ACM SIGIR conference on research and development in information retrieval, ACM Press, New Orleans, pp. 250-257 